\title{
Normalised Heart Rate Variability After Sacubitril/Valsartan
}

\author{
Simon Gerhardt ${ }^{1}$ and Joachim R Ehrlich ${ }^{1,2}$ \\ 1. Division of Cardiology, St Josefs-Hospital, Wiesbaden, Germany; 2. Division of Cardiology, Goethe University, Frankfurt, Germany
}

DOl: https://doi.org/10.17925/EJAE.2019.5.1.60

W present the case of a patient with heart failure and reduced ejection fraction. After changing from candesartan to sacubitril/valsartan, we saw a normalised heart rate variability.

\section{Keywords}

Heart rate variability (HRV), heart failure, implantable cardioverter-defibrillator (ICD), sudden cardiac death.

Disclosures: Simon Gerhardt and Joachim R Ehrlich have nothing to declare in relation to this article. Review Process: Double-blind peer review.

Compliance with Ethics: All procedures were followed in accordance with the responsible committee on human experimentation and with the Helsinki Declaration of 1975 and subsequent revisions, and informed consent was received from the patient involved in this case study.

Authorship: All named authors meet the criteria of the International Committee of Medical Journal Editors for authorship for this manuscript, take responsibility for the integrity of the work as a whole and have given final approval for the version to be published.

Received: 8 October 2018

Accepted: 21 January 2019

Citation: European Journal of Arrhythmia \&

Electrophysiology. 2019;5(1):60-1

Corresponding Author: Joachim R Ehrlich, Division of Cardiology, St Josefs-Hospital, Beethovenstr. 20, 65189 Wiesbaden, Germany. E: joachimehrlich@t-online.de

Support: No funding was received in the publication of this article.
We report the case of a 44-year-old woman with a history of chronic heart failure with reduced ejection fraction (HFrEF) due to ischaemic cardiomyopathy (New York Heart Association [NYHA] class II). In 2010, she had a myocardial infarction with thrombotic occlusion of the left anterior descending coronary artery, alleged in the context of an essential thrombocythaemia. The patient had an ejection fraction of 35\% and received a primary prevention subcutaneous defibrillator. Holter monitoring documented reduced heart rate variability (HRV) with a standard deviation of the normal-to-normal interval (SDNN) of $82 \mathrm{~ms}$ (Figure 1). The patient was switched to sacubitril/valsartan for prognostic purposes. After 4 months of angiotensin receptor/neprilysin inhibitor (ARNi) therapy we documented a normalised SDNN (162 ms, Figure 2). Furthermore, the rate of premature ventricular contraction (PVC) was greatly reduced to $0.1 \%$ after ARNi (41 PVC of 74,480 QRS complexes) compared to 1.7\% (1,030 PVC of 62,268 QRS complexes) before. Interestingly, the left ventricular systolic function did not change.

\section{Discussion}

Sacubitril/valsartan is indicated for treatment of symptomatic HFrEF. Its market approval was based on results of the pivotal PARADIGM-HF (Prospective Comparison of ARNi with ACEI to Determine Impact on Global Mortality and Morbidity in Heart Failure) study. This trial included 8,442 patients and was terminated early as it demonstrated a greater benefit of sacubitril/valsartan compared to enalapril in decreasing cardiovascular mortality and hospitalisations for heart failure. ${ }^{1}$

Sacubitril/valsartan reduced cardiovascular mortality through a similar reduction in pump failure and sudden cardiac death (heart rate 0.80; 95\% confidence interval; 0.73-0.87; $p<0.001$ ). ${ }^{1}$ Previous studies showed a correlation between myocardial wall stress with elevated natriuretic peptide and ventricular arrhythmias.,3 De Diego et al. recently demonstrated a reduction of ventricular arrhythmias in 120 patients with implantable cardioverter-defibrillator (ICD) and HFrEF largely due to ischaemic origin (82\%). After 9 months of therapy with conventional renin-angiotensin-aldosterone system inhibition, patients were switched to sacubitril/valsartan. ${ }^{4}$ This study found reduced incidence of ventricular arrhythmias and demonstrated an increase in left ventricular ejection fraction by $8 \%$. Natriuretic peptides may contribute to a reduction in sympathetic tone, and HRV is impaired in patients with HFrEF at risk of sudden cardiac death. ${ }^{5-7}$ Future studies will need to address the value of ICD implantation on top of optimal medical therapy with sacubitril/valsartan. ${ }^{8}$

Our case is the first report of an increase in HRV after therapy with sacubitril/valsartan. Thus, the development of HRV in our patient emphasises the potential beneficial effect of ARNi regarding a risk of sudden cardiac death. The fact that these findings are independent from the left ventricular function underpins this assumption. $\square$ 
Figure 1: Circadian heart rate and RR interval histogram of the Holter electrocardiogram prior to sacubitril/valsartan

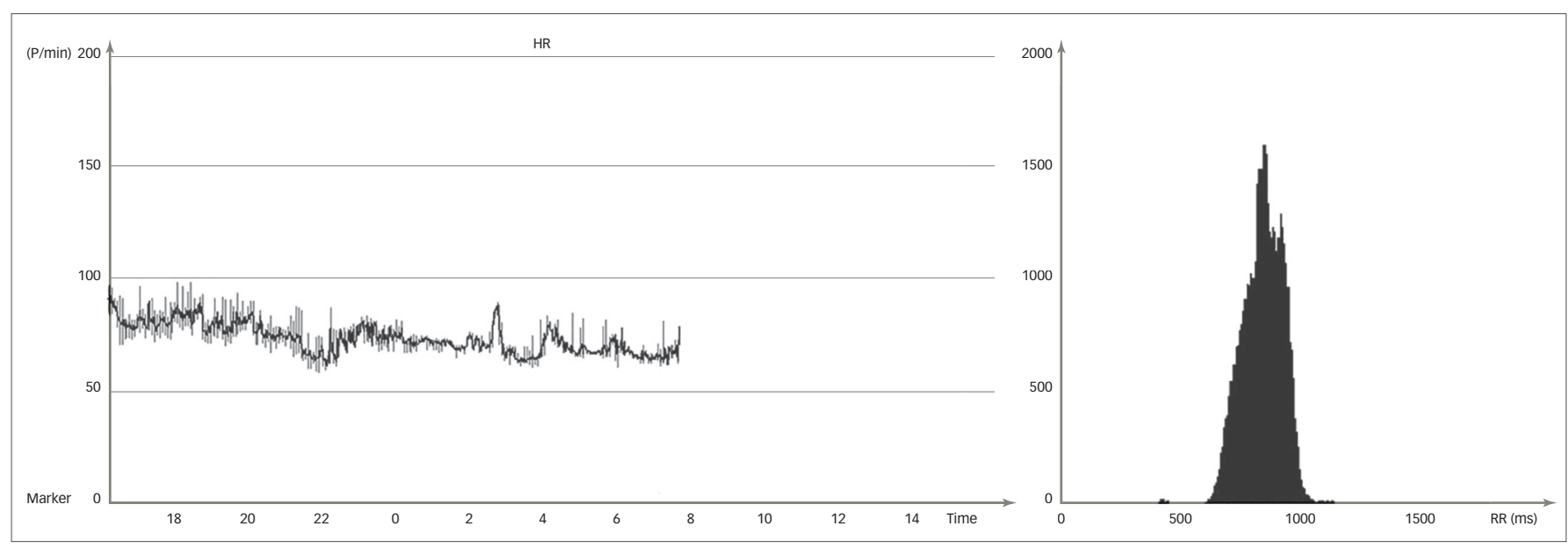

Standard deviation of the normal-to-normal interval $=82 \mathrm{~ms}$.

Figure 2: Circadian heart rate and RR interval histogram of the Holter electrocardiogram after sacubitril/valsartan

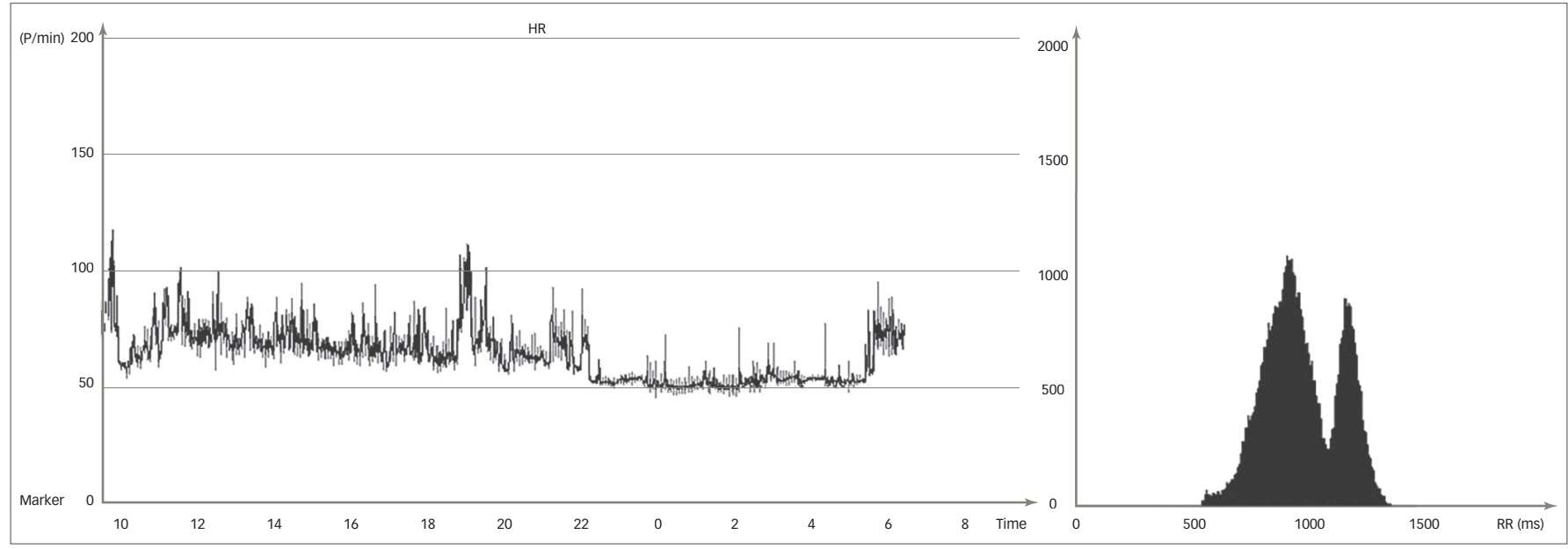

Standard deviation of the normal-to-normal interval $=162 \mathrm{~ms}$.

1. MCMurray JJ, Packer M, Desai AS, et al. Angiotensin-neprilysin inhibition versus enalapril in heart failure. $N$ Eng/ I Med. 2014:371:993-1004

Trayanova NA, Constantino J, Gurev V. Models of stretch-activated ventricular arrhythmias. J Electrocardiol. 2010:43:479-85.

Zabel M, Portnoy S, Franz MR. Effect of sustained load on dispersion of ventricular repolarization and conduction dispersion of ventricular repolarization and conduction
time in the isolated intact rabbit heart. I Cardiovasc Electrophysiol. 1996;7:9-16.
4. de Diego C, Gonzalez-Torres L, Nunez JM, et al. Effects of angiotensin-neprilysin inhibition compared to angiotensin inhibition on ventricular arrhythmias in reduced ejection fraction patients under continuous remote monitoring of implantable defibrillator devices. Heart Rhythm. 2018;15:395-402.

5. Kalla M, Herring N, Paterson DJ. Cardiac sympatho-vagal balance and ventricular arrhythmia. Auton Neurosci. 2016;199:29-37.
6. Bastiaenen R, Batchvarov V, Gallagher MM. Ventricular automaticity as a predictor of sudden death in ischaemic heart disease. Europace. 2012;14:795-803.

7. Kleiger RE, Miller JP, Bigger JT Jr, Moss AJ. Decreased heart rate variability and its association with inc meart rat veriablity and mortality atter acute myocard 1987

8. Ehrlich JR. Do we still need ICDs if we have ARNi? Heart Rhythm. 2018;15:403-4. 